\title{
Robust self-localisation and navigation based on hippocampal place cells
}

\author{
Thomas Strösslin *, Denis Sheynikhovich, Ricardo Chavarriaga, Wulfram Gerstner \\ Laboratory of Computational Neuroscience, Brain and Mind Centre, EPFL, 1015 Lausanne, Switzerland
}

\begin{abstract}
A computational model of the hippocampal function in spatial learning is presented. A spatial representation is incrementally acquired during exploration. Visual and self-motion information is fed into a network of rate-coded neurons. A consistent and stable place code emerges by unsupervised Hebbian learning between place- and head direction cells. Based on this representation, goal-oriented navigation is learnt by applying a reward-based learning mechanism between the hippocampus and nucleus accumbens. The model, validated on a real and simulated robot, successfully localises itself by recalibrating its path integrator using visual input. A navigation map is learnt after about 20 trials, comparable to rats in the water maze. In contrast to previous works, this system processes realistic visual input. No compass is needed for localisation and the reward-based learning mechanism extends discrete navigation models to continuous space. The model reproduces experimental findings and suggests several neurophysiological and behavioural predictions in the rat.
\end{abstract}

(C) 2005 Elsevier Ltd. All rights reserved.

Keywords: Spatial learning model; Hippocampus; Place cells; Head direction cells; Path integration; Calibration and drift removal; Nucleus accumbens; Reinforcement learning in continuous space

\section{Introduction}

The striking discovery of place cells in the rat hippocampus (O'Keefe \& Dostrovsky, 1971) has triggered a wave of interest on spatial learning that holds until today. The activity of place cells is highly correlated with the spatial location of the animal. Therefore, the hippocampus has been suggested to mediate spatial coding (O'Keefe \& Nadel, 1978; Redish, 1999). Furthermore, neurons whose activity is tuned to the orientation of the rat's head in the azimuthal plane (head direction cells) have also been found in the hippocampal formation (Ranck, 1984; Taube, Muller, \& Ranck, 1990). It is hypothesised that place and head direction cells together form a neural circuit for spatial representations (Knierim, Kudrimoti, \& McNaughton, 1995). Extensive experimental evidence suggests that two types of highly processed sensorial input influence the activity of the location and direction sensitive cells in the hippocampal formation: First, allothetic, i.e. external (visual, olfactory, tactile) sensory input from cortical areas is available for processing. It appears that visual information exerts a

\footnotetext{
* Corresponding author. Address: Neural Computation Unit, Okinawa Institute of Science and Technology, 12-22 Suzaki, Uruma, 904-2234 Okinawa, Japan.

E-mail address: thomas.strosslin@a3.epfl.ch (T. Strösslin).
}

0893-6080/\$ - see front matter (C) 2005 Elsevier Ltd. All rights reserved. doi:10.1016/j.neunet.2005.08.012 dominant influence compared to other modalities (Muller \& Kubie, 1987; Taube et al., 1990). Persistent location and direction sensitivity in the absence of external stimulation suggests that integration of idiothetic, i.e. internal selfmotion signals is another source of information processed by the neurons in the hippocampal formation (Jeffery, 2003; McNaughton et al., 1996). Experimental data shows that the activities of place and head direction cells correlate with behavioural decisions (see Jeffery, 2003 for review) and that lesions of related brain areas impair performance in navigational tasks where an internal space representation is necessary.

\subsection{Relation to our previous models}

This work presents a biologically inspired computational model of the rat's hippocampus and its role in navigation to a hidden goal location. It is based on a series of models by Arleo, et al. (Arleo, Smeraldi \& Gerstner, 2004; Arleo \& Gerstner, 2000b; Arleo, Smeraldi, \& Hug, 2001). As in the previous versions, an unsupervised growing network scheme is used. The model consists of interconnected populations of neurons that process allothetic and idiothetic inputs to drive the firing of simulated place cells. Visual input is represented by twodimensional gray-scale images. Relevant information about current position and gaze direction is extracted from the visual input to form allocentric location and direction estimates. Selfmotion input in the form of egocentric rotation and 
displacement signals are transformed to an arbitrary allocentric frame of reference and integrated into a position and heading estimate. The visual and self-motion processing pathways are then combined to form a spatial representation consisting of a large population of place cells with overlapping receptive fields. The synaptic efficacies between neural populations change according to Hebbian-type learning rules. Thus without any prior knowledge, the space code is built incrementally and on-line from direct interactions with the environment. This spatial representation is subsequently used for goal navigation using reinforcement learning. The state space is represented by the place cell population activity and locomotor neurons in nucleus accumbens, a target structure of the hippocampus, serve as action space.

The present model differs from previous models by Arleo et al. in several ways. The most important improvements are: (i) The new visual system closer emulates the visual system of the rat in that it has a wider view field of up to $320^{\circ}$ (Hughes, 1977). We also propose a uniform visual filter grid in contrast to a foveal retina. It is this rectangular grid which makes heading discrimination much simpler. (ii) The ability to reliably extract direction information from complex visual input removes the need of a polarising cue (a lamp) in order to calibrate the directional system. (iii) The former models explicitly access the spatial variance of place cell activity in order to recalibrate path integrator. This model combines visual and self-motion information in a simpler and more natural way. (iv) In previous models of learning goal-oriented behaviour, movements were restricted to four predefined directions. Just adding more directions would have resulted in an increased learning time due to the lack of generalisation in action space. The new model features a continuous action space allowing arbitrary directions of movement. A generalisation mechanism decouples the learning speed from the population size.

\subsection{Relation to other models}

The hippocampus was modelled extensively during the last decades due to its undisputed importance in memory and spatial behaviour. Here, we review previous work of other researchers which is relevant for this paper. The features of each model are briefly outlined and compared to our proposal. Specific similarities and differences are also mentioned in Sections 3 and 4. A summary of our contributions is given in Section 6.

Burgess et al. (Burgess, Recce, \& O'keefe, 1994) offer a detailed model of hippocampal place cells. It consists of several populations, representing entorhinal cortex (EC), hippocampus proper (HPC) and subiculum (SUB). Visual input is based on algorithmically calculated distances to landmarks placed near the walls. Neurons in SUB as well as head direction (HD) cells project to goal cells (GC). Rewardbased learning is applied for navigation. This model is also implemented on a robotic platform (Burgess, Donnett, Jeffery, \& O’Keefe, 1997; Burgess, Jackson, Hartley, \& O'Keefe, 2000; Hartley, Burgess, Lever, Cacucci, \& Keefe, 2000).
Unlike our proposal, their model uses simplified visual input (distance to the walls), which is not directly available to animals. It also suggests that the hippocampal formation is sufficient for locale navigation. This is in contradiction to experimental data where fornix lesions impair rats in the hidden water maze (Eichenbaum, Stewart, \& Morris, 1990; Packard \& McGaugh, 1992; Sutherland \& Rodriguez, 1990). Furthermore, their model fails in the presence of local obstacles as well as in darkness. Finally, their learning mechanism suffers a 'distal reward' problem. On the other hand, it proposes an abstract geometrical mechanism which produces the effect of phase precession. Our model does not address this issue.

The model proposed by Sharp et al. (Brown; Sharp, 1991 \& Sharp, 1995) builds on visual cells that encode the agent's distance and bearing to several landmarks in the environment. Subsequent layers, representing EC and HPC, combine visual information in order to form a place code. A population of 'motor' cells in nucleus accumbens receives spatial information from the hippocampal place cells. Together with a HD system, the model learns to perform movement commands which lead to a rewarding location. Their model relies on an artificial local view system. In contrast, our system uses realistic camera images. Whereas our model results in a highly redundant and distributed place code consistent with experimental data, their winner-take-all mechanism prevents all but one place cell from firing. Their representation has no path integration component and therefore does not support locationspecific firing in the dark. Our navigation system is similar to theirs. However, while their model needs a long temporal trace in order to overcome a distal reward problem, our approach does not suffer such a limitation. On the other hand, their model produces omnidirectional place fields in open, and unidirectional fields in an eight-arm-maze, consistent with experimental data. Our model's place fields are always omnidirectional.

The system by Redish et al. (Wan, Touretzky, \& Redish, 1994; Touretzky \& Redish, 1996; Redish and Touretzky 1997a,b) consists of separate populations for the local view, head direction, path integrator and place code. All populations interact with each other in order to form a consistent representation of space. Algorithmically determined information about type, distance and bearing angle to each landmark enters the local view system. The features of this place cell model are similar to ours. However, their neuronal model is more abstract. Their system relies on an abstract visual input, which features a perfect measure of the landmark type, distance and bearing. Our model extracts low-level features from real images. Place units in their model compute a 'fuzzy conjunction' of inputs in which terms that are unavailable or thought incorrect drop out. In contrast, we use standard neural activation function. Finally, unlike our proposal, there is no purely allothetic place code.

Hippocampal place cells in Abbott and colleagues' work (Blum \& Abbott, 1996; Gerstner \& Abbott, 1997) have perfectly Gaussian tuned receptive fields prior to navigation learning. Spike timing dependent plasticity on hippocampal 
collateral synapses results in a goal-dependent shift of place fields. This shift can be used for navigation. Multiple goal locations can be represented simultaneously. Whereas our model constructs a hippocampal place code from allothetic cues, their approach assumes perfectly Gaussian tuned place cells initially. In order to use the shifted place cells for navigation, they need to explicitly compare the initial and current place field centres. In our model, positions and actions are implicitly coded. Their model of locale navigation is entirely concentrated in the hippocampus. This conflicts with experimental data showing that lesions to the fornix or nucleus accumbens impair locale navigation (Eichenbaum et al., 1990; Packard \& McGaugh, 1992; Sutherland \& Rodriguez, 1990). On the other hand, their model can store navigation maps to multiple goal locations at the same time whereas ours is limited to one. Their neuron model and learning rule are also more detailed and realistic than ours.

The model proposed by Foster, Morris, and Dayan (2000) is based on an actor-critic architecture for temporal-difference reinforcement learning. A layer of place cells with perfectly tuned Gaussian receptive fields provides the navigation system with the agent's position within its environment. An actor network learns selecting an appropriate direction of movement, depending on place cell activity. In order to overcome interference with previously learnt goal locations, their model features a coordinate system module which learns to transform place code activity into a Cartesian frame and stores the goal's coordinates. Once learnt, algorithmic vector subtraction replaces the action selection based on learnt place/action associations. Whereas our place fields are learnt by experience, their model relies on a population of perfectly Gaussian tuned place cells with no allothetic component. Like our proposal, reinforcement learning is used between place cells and a set of action neurons which code for the allothetic direction of movement. Unlike our approach, however, learning does not generalise to similar directions and does not allow for continuous directions of movement in their system. On the other hand, their coordinate system enables the agent to quickly adapt to a new goal location. Our model does not address the quick relearning of a goal location. However, their coordinate-system module creates a global basin of attraction. Local obstacle avoidance is then no longer possible. Furthermore, the direction of movement is algorithmically calculated by explicitly accessing the coordinates of the goal location.

In the model by Gaussier et al. (Gaussier, Leprêtre, Joulain, Revel, Quoy, \& Banquet, 1998; Gaussier, Joalin, Banquet, Leprêtre, \& Revel, 2000; Gaussier, Revel, Banquet, \& Babeau, 2002), a local view is extracted from panoramic twodimensional images and consists of a set of landmark bearings and types. A landmark bearing is determined by a magnetic compass and its type is chosen from a predefined set. A place cell fires according to the similarity of the local views. A population of transition cells stores all transitions from each location (coded by a place cell) experienced in the past. This model relies on a compass and object recognition whereas ours works with low-level filter responses and without a compass.
Furthermore, this model uses winner-take-all mechanisms in all populations whereas we use a more biologically plausible distributed place code.

Being similar to these models in terms of the overall spatial coding procedure (i.e. storage and comparison of the local views) our model has the advantages of (i) working with realistic visual input, (ii) having an integrated head direction system which does not use any prior landmark information and (iii) a navigational system which can handle a continuum of possible actions, does not use any prior information about the environment and is able to work in the presence of obstacles as well as in darkness.

\section{Methods}

Our model consists of several interacting populations of 'rate-coded neurons'. This artificial neural network is synchronised by a global clock signal. During motion, a 6-12 Hz EEG oscillation called theta rhythm can be observed in the hippocampal formation of rats (Burgess et al., 1994; O'Keefe \& Recce, 1993; Skaggs, McNaughton, Wilson, \& Barnes, 1996). Strong inhibitory input from the septal region to the hippocampus seems to be responsible for generating these oscillations (Buzsáki, 1984; Hasselmo \& Bower, 1993; Miller, 1991; Winson, 1978). Theta is also observable during sensory scanning (e.g. sniffing). We assume that theta serves as a clock signal to synchronise information processing throughout the hippocampal formation.

\subsection{Model architecture and biological background}

The input layers of our model process raw sensory input (visual images and odometric information). Intermediate layers encode allocentric estimations of position and direction and the output layer controls goal-oriented locomotor actions of the agent. The model architecture is shown in Fig. 1. Labels to the left of populations denote function, whereas the candidate brain area is indicated to the right. We first introduce the shorthand notation used throughout this paper and then continue with some experimental evidence supporting our choice of neural substrates. Next, we summarise and justify the use of reinforcement learning for navigation and explain why we choose the Morris water maze as validation task.

The local view (LV) processes, stores and compares visual stimuli. We model two types of local view cells: rotation cells (RCs) and step cells (SCs). SCs drive the allothetic place code (APC), located in lateral entorhinal cortex (IEC). RCs calibrate the head direction system (HD) in the postsubiculum (poSb). Idiothetic input drives the HD and the position integrator (PI), located in the medial entorhinal cortex. APC calibrates PI and they both project to the combined place code (CPC) of the hippocampus HPC and possibly subiculum. The CPC is used for navigation learning on directional action cells (ACs) in nucleus accumbens (NA).

The interconnections between these neural populations correspond to anatomical findings in the rat (Amaral, \& Witter, 1989, 1995). Extensive lesion studies show that damage to 


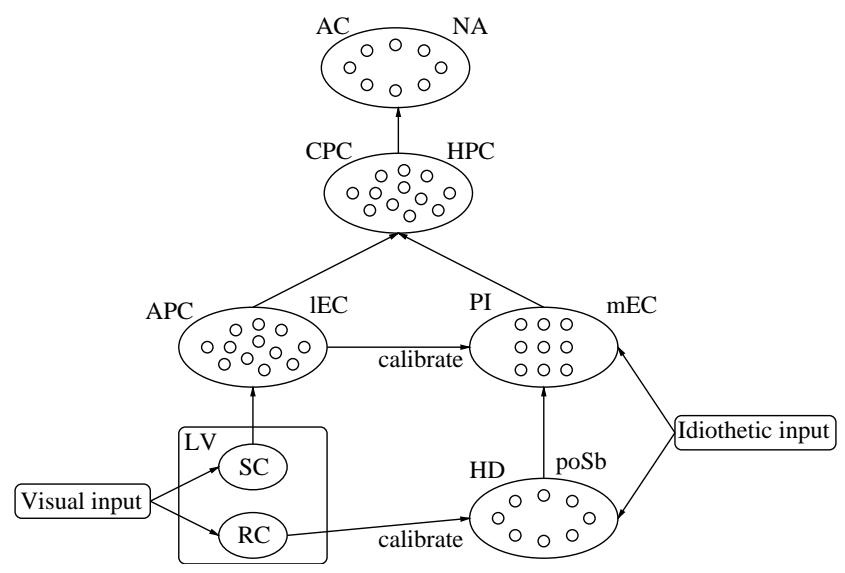

Fig. 1. Model architecture. The local view (LV), embracing rotation cells (RCs) and step cells (SCs), processes, stores and compares visual stimuli. SCs drive the allothetic place code (APC) in the lateral entorhinal cortex (IEC) and RCs calibrate the head direction system (HD) in the postsubiculum (poSb). Internal odometric input drives HD and the position integrator (PI) in medial entorhinal cortex. APC calibrates PI and they both project to the combined place code (CPC) in the hippocampus (HPC) and subiculum. CPC is used for navigation learning on directional action cells (ACs) in nucleus accumbens (NA).

brain areas containing place or direction sensitive cells, as well as lesions of NA selectively impair rats in the hidden platform water maze task (Packard, \& McGaugh, 1992; Redish, 1999).

Additionally, NA seems to be involved in rewarddependent learning of spatial locomotion (Whishaw, \& Mittleman, 1991). Several neurophysiological properties of biological neurons in the hippocampus of the rat are captured by the artificial place cells that develop in the model in an unsupervised manner as a result of environment exploration. The activity of the location sensitive neurons in CA3-CA1 areas of the hippocampus are shown to depend strongly on distal visual stimuli (see (Redish, 1999) for an excellent review). The activity of hippocampal neurons is shown to be direction independent in an open field (Markus et al., 1994; McNaughton, Barnes, \& O'Keefe, 1983; Muller, Bostock, Taube, \& Kubie, 1994; O’Keefe, \& Burgess, 1996). The location sensitive firing persists in the absence of visual stimulation, i.e. when the lights are turned off after exploration (Quirk, Muller, \& Kubie, 1990; Save, Nerad, \& Poucet, 2000). This suggests that integration of idiothetic cues influence place cells. Place cells in mEC preserve firing topology across reshaped environments. mEC cells are also likely to be active in any environment (Quirk, Muller, Kubie, \& Ranck, 1992). This indicates that mEC might be part of a general, environment-independent position integration system. Little is known, however, about the lateral part of entorhinal cortex. Electrophysiological studies are lacking for this region. Lesion studies suggest, however, that IEC is involved in the encoding of allothetic sensory signals (Otto, Ding, Cousens, \& Schiller, 1996). As this is the only other pathway to HPC, we postulate that the lateral entorhinal cortex contains an environment-dependent allothetic place code. There is also evidence for strong synaptic innervation from the lateral to the medial region of EC (Quirk et al., 1992). These could convey allothetic spatial information used to calibrate a position integrator in mEC. Cells which respond to the allocentric head direction of rats have been found in poSb (Ranck, 1984; Taube et al., 1990). They seem to be heavily influenced by idiothetic and visual stimuli.

One of the existing hypotheses of how place cells can be used for navigation employs a reinforcement learning framework in order to associate place with goal information. In the reinforcement learning theory (Sutton, \& Barto, 1998), a mapping between a state space (e.g. place cells) and an action space (e.g. locomotor cells) is learnt via the estimation of a state-action value function. The value function corresponds to the expected future reward and can be learnt on-line by trial and error based on the difference between the predicted and an actual reward. It was found that the activity of dopaminergic neurons in the basal ganglia is related to the errors in reward prediction (Schultz, 1998; Schultz, Dayan, \& Montague, 1997). Furthermore, these neurons project to NA, on the same spines as hippocampal afferents (Freund, Powell, \& Smith, 1984; Sesack, \& Pickel, 1990).

The hidden platform water maze task (Morris, 1981; Redish, 1999) is a frequently used paradigm to test navigation capabilities. The experimental setup consists of a circular water pool filled with opaque liquid and a small platform located inside the pool and submerged below the surface of the liquid. At the beginning of each trial, a rat is placed into the pool at a random location and its task is to find the platform. Since no visual cues directly identify the platform and the starting locations are random, animals have to remember the location of the hidden platform based on the extra-pool visual features. After several trials, rats are able to swim directly to the hidden platform from any location in the pool. This task is known to depend on an intact hippocampus. We therefore choose this paradigm as a validation of our model.

\subsection{Experimental setups}

In order to validate our model, we also implement it on a computer, which is connected to a Khepera ${ }^{1}$ mobile robot (Mondada, Franzi, \& Ienne, 1994). The Khepera is equipped with a camera, odometers and proximity sensors (cf. Fig. 2(a)). Additionally, we use a simulated Khepera robot in virtual environments for our test experiments. The robot is controlled by the neural model and monitored by a tracking system. We use the term 'agent' throughout this article when we do not differentiate between the real and the virtual robot. Both emulate a rat in an experimental arena.

The experiments presented in Section 5 are run in four different configurations. All of them are static throughout the experiment:

Office: This setup consists of the real Khepera robot placed in an $80 \times 60 \mathrm{~cm}$ arena on a table in a normal office

\footnotetext{
${ }^{1}$ The Khepera mobile robot manufactured by K-Team (http://k-team.com/) is a modular platform popular for research and education.
} 


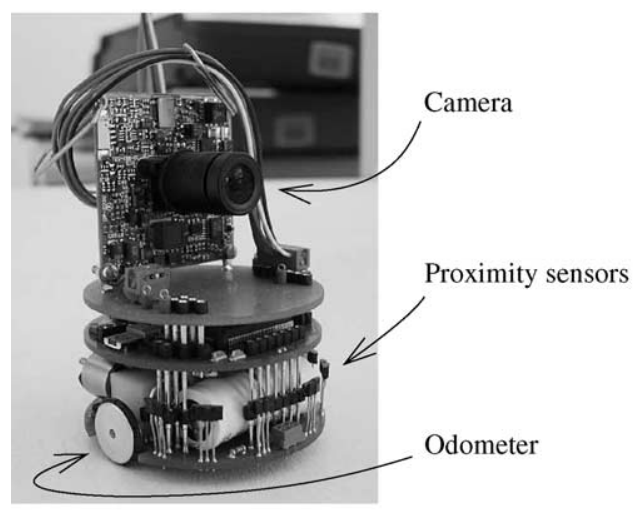

(a)

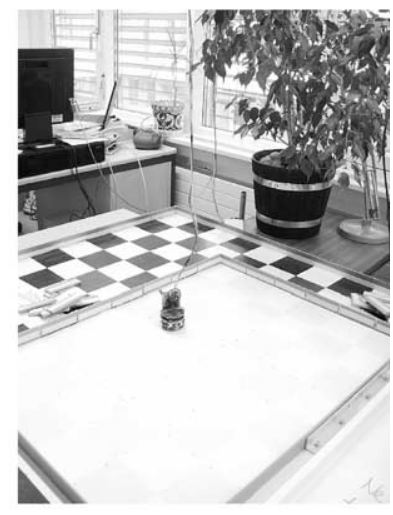

(b)

Fig. 2. (a) The Khepera robot and its sensors. (b) View of the 'Office' environment with the Khepera robot.

(cf. Fig. 2(b)). The arena is surrounded by borders of $3 \mathrm{~cm}$ height. The Khepera's camera has a viewangle of approximately $60^{\circ}$ (see Fig. 2). Four pictures in directions separated by $60^{\circ}$ are merged into a single image $\mathscr{I}$ of approximately $\theta=240^{\circ}$ by rotating the robot in-place. Note that these rotations are only performed in order to acquire a panoramic view.

Buildings: This and the following environments emulate the Khepera robot in a $77 \times 77 \mathrm{~cm}$ virtual world. Images are pasted to walls placed outside of the arena. The view of the virtual world is projected onto a cylindrical screen which covers a viewangle of $280^{\circ}$ using standard computer graphics algorithms. In the 'Buildings' setup, four walls are placed in a square around the environment and decorated with pictures of buildings and other manmade structures.

Davos: This natural scene contains less structure than the man-made objects of the previous two setups. Here, a panoramic view of the Swiss mountains is pasted onto a big cylinder surrounding the virtual arena.

Minimal: The previous environments all provide rich visual stimuli. In most animal experiments, however, the view is restricted to a small number of well-defined cues. In order to emulate such an impoverished environment, four walls are placed in a square around the arena. In the centre of each wall, one simple geometrical object is placed. The objects are a filled black square, a filled white circle, a triangle and a double cross. The background of each wall is covered with low contrast noise.

The agent constantly acquires panoramic $800 \times 316$ grayscale images of its environment during experiments. This highdimensional sensory input needs to be preprocessed before it is passed on to the neural network model. We use a mechanism which is inspired by neuronal properties in primary visual cortex: The image data is represented using a set of Gabor wavelets (Gabor, 1946). They respond to bars of different widths (spatial frequencies) and orientations in the image much like complex cells in visual cortex area V1 (Hubel, \& Wiesel, 1962). A filter vector $\vec{f}_{j}$ is calculated as the magnitude of the complex filter responses to the image. The filter responses are calculated on a set of sampling points in the image. These sampling points form an artificial rectangular retina. An example of the retinal response is shown in Fig. 3 for the 'Buildings' environment. The retina consists of 15 columns and 3 rows. On each point, the filter response for the lowest frequency (wavelength $\lambda_{j}=50$ pixels) is represented by a black

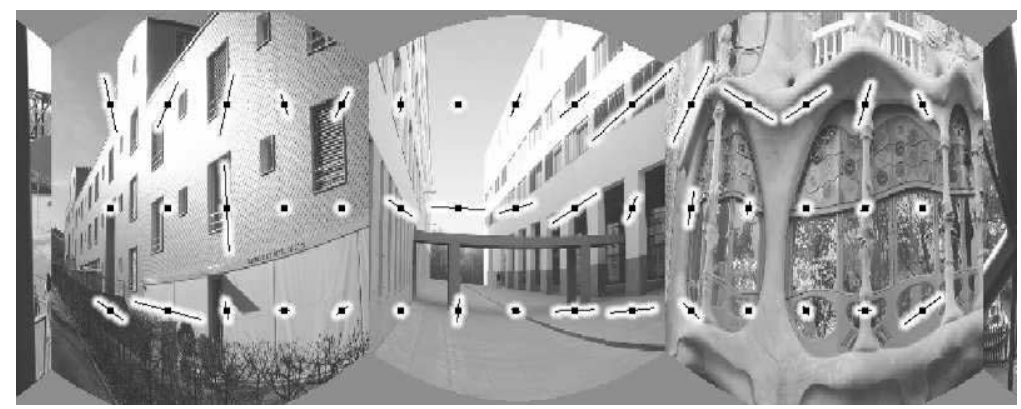

Fig. 3. Response of the artificial retina applied to a view from the 'Buildings' environment. The black lines represent the weighted sum of the gabor filter responses for all orientations. They indicate the direction and 'strength' (line length) of edges near each retinal point. 
line. It indicates the direction and 'strength' (line length) of edges in its neighbourhood.

\section{Spatial representation}

Similar to (Arleo \& Gerstner, 2000b; Burgess et al., 1994; Gaussier et al., 2000; Sharp, 1991; Touretzky, \& Redish, 1996), the architecture of our localisation system (Fig. 1) is inspired by experimental works on various levels (electrophysiology, anatomy, behaviour, etc.) in the rat. The model consists of five interconnected components: (i) The local view module (LV) stores and compares visual input in rotation cells (RCs) and step cells (SCs). (ii) The head direction system (HD) continuously updates the agent's sense of orientation. (iii) The allothetic place code (APC) estimates the agent's position within the environment based on the local view. (iv) The position integrator (PI) keeps track of the agent's location relying on odometric information. (v) The combined place code (CPC) links the visual and odometric position estimates and forms the output of the localisation system. It is used by the navigation module (cf. Section 4) to learn goal-oriented behaviours.

\subsection{Local view}

The local view module receives visual input from the columns of the artificial retina. Its purpose is to store and compare relevant information, for the construction of a spatial representation (Touretzky \& Redish, 1996). At each time step, several view cells are recruited and tuned to the current retinal response. We assume that there are enough recruitable neurons and do not address the question of 'resource management'. A previous view stored at time $t_{k}$ can be compared to the current view at time $t_{j}$ by defining a similarity measure between two sets of filter activities. This measure is implemented by visual neurons described in this section.

The choice of a comparison function depends on what transformations the image is subject to. The agent's local view in an environment depends on two variables, namely position and heading. Accordingly, we will call an agent's movement a 'rotation' if it only changes the heading. If only the agent's position is changed by a small amount, we call it a 'step'. Independently of the environment, a rotation produces a translation along the horizontal axis of the view. A step, however, causes a complicated and environment-dependent transformation. We assume that translations along the vertical axis are not possible, i.e. the agent does not look up or down, only left or right. While this holds for the mobile robot, it is only an approximation for the rat.

One of the problems is that if the visual cues are far away, a step produces only a small change in the view, whereas a rotation always produces a big, but predictable change. For a spatial representation, however, the visual system must permit the discrimination of both heading and position. We therefore 'read' the retinal information in two ways: One neural population tries to discriminate well between headings but not position, and vice versa for a second population. Both should have broad tuning curves in order to provide good generalisation of known samples to new views.

\subsubsection{Rotation cells}

Rotation cells (RCs) aim at discriminating headings regardless of position. The receptive fields should span a large range of headings, i.e. moderate translations of the image should not cause a drastic change in activity.

This is achieved by combining information from neighbouring retinal columns. For each retinal column i positioned at $x_{i}$, the weighted sum of filter vectors $\vec{h}\left(x_{i}, t_{k}\right)$ at time $t_{k}$, not the filter vectors $\vec{f}\left(x_{i}, t_{k}\right)$ themselves, are stored / compared:

$\vec{h}\left(x_{i}, t_{k}\right)=c_{0} \cdot \vec{f}\left(x_{i}, t_{k}\right)+\sum_{j=1}^{\left[n_{\text {cols }} / 2\right]} c_{j} \cdot\left[\vec{f}\left(x_{\operatorname{left}(i, j)}, t_{k}\right)+\vec{f}\left(x_{\operatorname{right}(i, j)}, t_{k}\right)\right]$

where left $(i, j)=|i-j|$ and right $(i, j)=\left(n_{\text {cols }}-1\right)-\mid i+j-$ $\left(n_{\text {cols }}-1\right) \mid$ are the $j$ th column indices to the left and right of the current column $i$. In the centre, left $(i, j)=i-j$ and right $(i, j)=$ $i+j$. Near the borders, however, columns which would lie outside of the image are mirrored on the left and right borders. Note that vector $\vec{f}$ in Eq. (1) is composed of the filter responses from all rows of column $i$. The weights $c_{j}$ are sampled from a Gaussian $\mathcal{N}_{c}$, the standard deviation $\sigma_{c}=100$ pixels of which determines the amount of translation invariance.

In order to compare two views, a similarity measure must be defined. At each time step $t_{k}$, the agent takes a visual snapshot. The image is processed as described above and encoded by a set of RCs. For each column $i$, a newly recruited RC stores the feature vector $\vec{h}\left(x_{i}, t_{k}\right)$. The activity $\mathrm{r}\left(t_{j} \mid x_{i}, t_{k}\right)$ of an RC represents the similarity of a column at time $t_{j}$ with respect to what the cell stored at time $t_{k}$. The activation function is:

$r\left(t_{j} \mid x_{i}, t_{k}\right)=\exp \left[-\left(\left\|\vec{h}\left(x_{i}, t_{k}\right) \ominus \vec{h}\left(x_{i}, t_{j}\right)\right\|_{1}\right)^{2} /\left(2 k \cdot \sigma^{2}\right)\right]$

where $\vec{z}=\vec{x} \ominus \vec{y}$ is the relative difference: Each element $l$ of the normal difference is element-wise divided by $\vec{x}$, i.e. $z_{l}=\left(x_{l}-y_{l}\right) / x_{l}$. This is somewhat similar to a shift and scale of the input distribution and allows sensory input from statistically different environments be treated in the same way, thus simulating sensitivity adaptation or habituation. $\|\cdot\|_{1}$ denotes the L1-norm. $k=488$ is the product of the number of rows per retina column and the number of gabor frequencies and orientations. $\sigma=0.25$ is a hand-tuned constant. Thus, $r$ depends on the average relative distance between stored and current filter activity.

\subsubsection{Step cells}

The second way of reading out the retinal activation helps discriminating positions, regardless of the agent's heading. Suppose that two distinguishable landmarks $l_{1}$ and $l_{2}$ are the only visual cues in an environment. Let us define $\phi(\boldsymbol{P})$ as the difference in the bearing angle to landmarks $l_{1}$ and $l_{2}$ when the agent is at location $\boldsymbol{P}$. If the agent has a field of view of $360^{\circ}$, the two landmarks are visible for all agent headings 
and the difference $\phi(P)$ is independent of this heading. However, the bearing difference depends on the agent's position, i.e. for most positions $P_{1}$ and $P_{2}, \phi\left(P_{1}\right) \neq \phi\left(P_{2}\right)$. Similar to (Touretzky, \& Redish, 1996), this property is exploited by the step cells (SCs). However, we do not really use landmarks, but low-level Gabor filters.

At every time step $t_{k}$, a variable number of SCs is recruited: We consider two retinal columns $s$ and $s+\delta$. If the filters of both columns are sufficiently active, their filter vector difference $\vec{d}\left(\delta, s, t_{k}\right)$ is stored if:

$\delta \in\{3, \ldots, 6\}$ and $\left\|\vec{f}\left(x_{s}, t_{k}\right)\right\|_{1}>\theta_{\text {act }}<\left\|\vec{f}\left(x_{s+\delta}, t_{k}\right)\right\|_{1}$

$\vec{d}\left(\delta, s, t_{k}\right)=\vec{f}\left(x_{s}, t_{k}\right)-\vec{f}\left(x_{s+\delta}, t_{k}\right)$

The empirically determined activity threshold $\theta_{\text {act }}=1.0$ is particularly useful in impoverished environments where most filter columns just see plain walls. It prevents recruitment of cells that store no relevant information.

The column distance $\delta$ should ideally correspond to big angle differences because-at least in enclosed environments-big landmark bearing differences depend more on the agent's position than small ones, which makes position discrimination easier. However, if the agent's field of view $\Psi$ is smaller than $360^{\circ}$, a big value for $\delta$ reduces the probability that both landmarks are visible at the same time. In our experiments, $\Psi$ equals $280^{\circ}$. We allow a range of empirically determined column distances $\delta \in\{3, \ldots, 6\}$. This corresponds to bearing differences between approximately $50^{\circ}$ and $100^{\circ}$.

SCs should detect at time $t_{j}$ if the difference of column filter activities of a given distance $\delta$ has already been seen and stored at time $t_{k}$. The comparison should be independent of the agent's heading, i.e. the absolute position of the two columns spaced by $\delta$ on the retina should not matter. To achieve this translation invariance in the retinal image, $\vec{d}\left(\delta, s, t_{k}\right)$ is compared with the most similar column difference at time $t_{j}$. The firing rate $r\left(\mathrm{t}_{\mathrm{j}} \mid \delta, s, t_{k}\right)$ of an SC which stored the difference vector $\vec{d}\left(\delta, s, t_{k}\right)$ at time $t_{k}$ is given by:

$r\left(t_{j} \mid \delta, s, t_{k}\right)=\exp \left[-\left(\min _{i}\left\|\vec{d}\left(\delta, s, t_{k}\right) \ominus \vec{d}\left(\delta, i, t_{j}\right)\right\|_{1}\right)^{2} /\left(2 k \cdot \sigma^{2}\right)\right]$

where $k$ is the same normalisation factor as for RCs (Eq. (2)) and $\sigma=0.1$ is a hand-tuned constant.

\subsection{Head direction system}

The head direction (HD) module forms the most important part of the spatial learning model. Small rotational errors can totally impair the system's ability to build and maintain a spatial representation. This section describes how the HD system combines odometric and visual information in order to produce a stable (non-drifting) estimate of the agent's heading. Similar to (Arleo, \& Gerstner, 2001), a population of $N_{\text {hd }}=120$ directional neurons codes for the agent's heading $\Phi$ with respect to an arbitrary fixed compass bearing $\Psi$. Without loss of generality, we assume $\Psi=0$. Each HD neuron $i$ represents the heading $\phi_{i}=i \cdot 360^{\circ} / N_{\mathrm{hd}}$.

The firing rates of HD cells is calculated in two stages. First, an estimation $\Phi_{\mathrm{hd}}$ of the agent's real heading $\Phi$ is calculated as described below. Then, a large activity profile around $\Phi_{\mathrm{hd}}$ is enforced in the HD system. Lateral interconnection between HD cells could be the neuronal substrate for such activity profiles (Boucheny, Brunel, \& Arleo, 2005; Zhang, 1996). Here, we emulate lateral interactions by enforcing a Gaussian activity profile around $\Phi_{\text {hd }}$. Formally, the firing rate $r_{i}$ of HD cell $\mathrm{i}$ is

$r_{i}=\exp \left[-\left(\left\|\Phi_{\mathrm{hd}}-\phi_{i}\right\|_{\varphi}\right)^{2} /\left(2 \sigma^{2}\right)\right]$

where $\|\cdot\| \varphi \in[0,2 \pi]$ is the angular distance and $\sigma^{2}$ is the angular variance of the Gaussian profile. In our experiments, a value of $\sigma=60^{\circ}$ is used.

When the agent moves ${ }^{2}$ at time step $t-1$, its angular displacement $\delta \Phi_{\mathrm{i}}(t-1)$ is estimated by dead-reckoning and made available to the HD system when the movement is completed. The new heading, estimated by idiothetic cues, is then $\Phi_{\mathrm{i}}(t)=\Phi_{\mathrm{hd}}(t-1)+\delta \Phi_{\mathrm{i}}(t-1)$.

Any real-world path or heading integration system based on dead-reckoning is subject to noise and, more importantly, to a systematic drift. In order to keep the HD representation accurate, the local views encountered during exploration are continuously associated to their heading direction. In particular, all rotation cells (RCs) are connected to all HD cells and synapses are activated/modified at each time step as follows: If an inactive synapse's pre- and postsynaptic activities $r_{i}$ and $r_{i}$ are above a threshold $\theta_{\text {act }}=0.2$, the synapse is activated and its strength initialised using a one-shot Hebbian-type learning rule:

$w_{i j}=r_{i} \cdot r_{j} \quad$ if $\quad r_{i}>\theta_{\text {act }}$ and $r_{j}>\theta_{\text {act }}$

Once activated, the synaptic efficacy is modified at each time step:

$\Delta w_{i j}=\eta \cdot r_{i}\left(r_{j}-w_{i j}\right)$

where $\eta=0.01$ is the learning rate. After each learning step, weights are renormalised: $\tilde{w}_{i j}=w_{i j} /\left(\sum_{k} w_{i k}\right)$. The activities of all RCs $j$ connected to HD cell $i$ produce an input potential $h_{i}=\sum_{j} \tilde{w}_{i j} r_{j}$ at the HD cell $i$. The allothetic heading estimate $\Phi_{\mathrm{a}}$ is then given as a circular 'population vector' of the input potentials $h_{i}$ :

$\Phi_{\mathrm{a}}=\arctan \left(\frac{\sum_{i} h_{i} \sin \left(2 \pi i / N_{\mathrm{hd}}\right)}{\sum_{i} h_{i} \cos \left(2 \pi i / N_{\mathrm{hd}}\right)}\right)$

Unlike the more general population vector decoding algorithms (Georgopoulos, Schwartz, \& Kettner, 1986; Salinas, \& Abbott, 1994; Sanger, 2003), this simple form does not need the place field distribution. On the other hand, it only works well for uniformly distributed symmetrical place cells. We still explicitly access angular information in Eqs. (5), (8) and (9) which seems at first sight biologically implausible. However, if

\footnotetext{
2 It is always assumed that the agent performs 'uniform' movements. The movements is uniform in the sense that the angular velocity $\omega$ remains constant throughout the movement.
} 
continuous attractors using lateral connections (Amari, 1977; Boucheny et al. 2005; Zhang, 1996) or a probabilistic activity transition matrix (Herrmann, Pawelzik, \& Geisel, 1999) between HD cells was used for implementing Eq. (5), the recalibration could be performed without explicitly accessing angular information. Furthermore, it has been shown that attractor dynamics combined with basis functions can find optimal multisensory integration (Deneve, Latham, \& Pouget, 2001; Pouget, Deneve, \& Duhamel, 2002).

At each time step, both idiothetic and allothetic heading estimations are determined. Unlike (Arleo, \& Gerstner, 2000a; Gaussier et al., 2000), our system performs calibration based only on real-world visual information. No compass or polarising cue is needed. The total estimated heading $\Phi_{\mathrm{hd}}$ is then calculated as:

$\Phi_{\mathrm{hd}}=\Phi_{\mathrm{i}}-\alpha \cdot\left(\Phi_{i}-\Phi_{\mathrm{a}}\right)$

$\alpha \in[0,1]$ determines the influence of the visual estimate. A value of zero means no visual influence at all whereas a value of one would completely ignore odometric information.

A nice property of the idiothetic update is that it is always smooth. There are no 'jumps' if the noise in the dead-reckoning system is not too big. This smoothness is not present in the visual heading estimation. At each time step, the previous heading is forgotten and a new independent estimation is performed. In other words: Odometric estimation contains memory and visual estimation does not. For this reason, $\alpha$ in Eq. (9) should be small. We use $\alpha=0.1$.

\subsection{Visual place cells}

In this component of the model, a spatial representation based on visual information is constructed from experience. Unlike (Burgess et al., 1994; Sharp, 1991; Touretzky, \& Redish, 1996), our system can deal with real-world input, much like (Arleo, Smeraldi, \& Gerstner, 2004; Gaussier et al., 2000). In each time step, a new allothetic place cell (APC) $i$ is recruited. As for the local view, we assume that there are enough recruitable cells. Neurons coding for the current local view synapse on the new place cell $i$. In particular, a step cell (SC) $j$ connects with weight $w_{i j}$ to place cell $i$ if its firing rate $r_{j}$ is higher than $\theta_{\text {act }}=0.8$ :

$w_{i j}=\left\{\begin{array}{cc}r_{j} \underbrace{\left(\cdot r_{i}\right)}_{=1} & \text { if } r_{j}>\theta_{\text {act }} \\ 0 & \text { else }\end{array}\right.$

This is a thresholded one-shot Hebbian-type rule with learning rate one. The newly recruited cell should represent the current place. Therefore, it should be maximally active $\left(r_{i}=1\right)$ for the current afferent SC projection. This is achieved by tuning the parameters of the neuron's piecewise linear activation function:

$$
r_{i}= \begin{cases}0 & \text { if } \kappa_{i} h_{i}<\theta_{\text {low }} \\ 1 & \text { if } \kappa_{i} h_{i}>1 \\ \left(\kappa_{i}\left(h_{i}-\theta_{\text {low }}\right) /\left(1-\theta_{\text {low }}\right)\right. & \text { else }\end{cases}
$$

where $h_{i}=\sum_{j} w_{i j} r_{j}$ is the input potential to APC neuron $i, \theta_{\text {low }}=$ 0.2 is the minimal input to activate the neuron and $\kappa_{i}=1 / h_{i}^{0}$ determines the saturation potential of neuron $i$, with $h_{i}^{0}$ standing for the input potential at the time when neuron $i$ was recruited. At the moment when the cell $i$ is recruited, we have $\kappa \cdot h_{i}=1$, and hence $r_{i}=1$.For this reason, $r_{i}$ may be omitted in Eq. (10).

The resulting place code represents the agent's position in the environment. For the same reasons as in the HD system, a simple formula is used for decoding the population activity:

$\mathbf{P}_{\mathrm{a}}=\frac{\sum_{i} r_{i} \cdot \mathbf{x}_{i}}{\sum_{i} r_{i}}$

where $\mathbf{x}_{i}$ is the agent's position where APC $i$ was recruited.

\subsection{Position integration}

The HD system described above keeps track of the agent's current compass bearing. The position integrator (PI) module presented in this section implements the memory for the agent's current location. Together, they work as a path integrator, i.e. an environment-independent spatial representation. The PI system is mainly driven by idiothetic input. Similar to (Arleo, \& Gerstner, 2000b; Redish, \& Touretzky, 1997a; Touretzky, \& Redish, 1996), allothetic information is used to initialise or recalibrate PI neurons.

A population of $N_{\mathrm{pi}}=400$ simulated neurons encode the agent's estimated position $\mathbf{P}_{\mathrm{pi}}$ in a Cartesian coordinate frame. Each PI neuron $j$ is assigned a predefined preferred position $\mathbf{p}_{j}$ such that a square region of space is uniformly covered. The firing rate $r_{j}$ of cell $j$ is a two-dimensional Gaussian with standard deviation $\sigma=45 \mathrm{~mm}$ over the euclidian distance $\left\|\mathbf{P}_{\mathrm{pi}}-\mathbf{p}_{j}\right\|_{2}$ :

$r_{j}=\exp \left[-\left(\left\|\mathbf{P}_{\mathrm{pi}}-\mathbf{p}_{j}\right\|_{2}\right)^{2} /\left(2 \sigma^{2}\right)\right]$

As with the HD system, such an activity profile may result from lateral interactions between the PI neurons. Similarly to the HD system, an idiothetic estimate $\mathbf{P}_{\mathrm{i}}$ is calculated using dead-reckoning (amount of displacement) as well as HD (direction of movement) information.

In order to prevent $\mathbf{P}_{\mathrm{i}}$ from drifting away, the representation needs to be recalibrated using allothetic information. At each time step, the idiothetic and allothetic position estimates $\mathbf{P}_{\mathrm{i}}$ and $\mathbf{P}_{\mathrm{a}}$ are determined. The new recalibrated position estimate $\mathbf{P}_{\mathrm{pi}}$ is then calculated as:

$\mathbf{P}_{\mathrm{pi}}=\mathbf{P}_{\mathrm{i}}-\beta \cdot\left(\mathbf{P}_{\mathrm{i}}-\mathbf{P}_{\mathrm{a}}\right)$

where $\beta \in[0,1]$ determines the influence of the allothetic estimate. Similar to the HD system, a value of zero means no allothetic influence and a value of one would completely ignore idiothetic information. We use $\beta=0.1$ in our experiments. As 
in the HD system, a small value of $\beta$ keeps the position estimate smooth, while still removing systematic drifts. Similarly to the HD system, we could associate allothetic place cell activity to the firing of idiothetic place cells using unsupervised Hebbian learning and use an attractor network for implementing Eq. (13). Then, no explicit spatial information would be needed for recalibration.

\subsection{Combined visual and path integration place cells}

The idiothetic and allothetic information converges in a layer of combined place code (CPC) neurons, much like (Arleo, \& Gerstner, 2000; Touretzky, \& Redish, 1996). At each time step, a new place cell is tuned. Synapses originating in the APC and PI layers are recruited and initialised as defined by Eq. (10). The firing rate of CPC neuron $\mathrm{i}$ is given by Eq. (11). However, the input threshold $\theta_{\text {low }}=0.3$ in the CPC layer is higher than for APC neurons. This results in a sparser representation and smaller receptive fields. Additionally, at each time step, weights $w_{i j}$ of $\mathrm{APC} \rightarrow \mathrm{CPC}$ synapses are modified using a Hebbian-type learning rule with learning rate $\eta=0.1$ :

$\Delta w_{i j}=\eta r_{i}\left(r_{j}-w_{i j}\right)$

where $r_{i}$ is the firing rate of CPC neuron $i$ and $r_{j}$ is the firing rate of APC neuron $j$.

\section{Learning a navigation map}

In this section, a new model of rodent navigation is presented. In particular, we propose a locale navigation model (Redish, 1999). This type of navigation allows the animal to learn to find a stable but hidden reward location in an environment. The navigation system is based on the spatial representation learnt according to Section 3. Similar to (Arleo, \& Gerstner, 2000b; Brown, \& Sharp, 1995; Burgess et al., 1994; Foster et al., 2000) and unlike (Blum, \& Abbott, 1996; Gaussier et al., 2002; Redish, \& Touretzky, 1998), a navigation map is learnt outside of the hippocampus. In particular, Q-learning, a reinforcement learning $(\mathrm{RL})$ variant (Sutton, \& Barto, 1998) is applied to the HPC $\rightarrow$ NA synapses (Arleo, \& Gerstner, 2000b; Brown, \& Sharp, 1995). RL has previously been used to solve navigation tasks for autonomous mobile agents (Arleo, \& Gerstner, 2000b; Arleo, Smeraldi, \& Gerstner, 2004; Brown, \& Sharp, 1995; Foster et al., 2000). Some models operate in continuous state and / or action spaces using function approximation. However, we are not aware of any neural model of locale navigation where both state and action spaces are continuous.

\subsection{Action cells}

A population of $N_{\mathrm{ac}}=120$ action cells (ACs) code for the motor-commands of the agent's next movement. Each AC $i$ represents a particular allocentric heading $\phi_{i}$, which are uniformly distributed between 0 and $2 \pi$. In each time step, the agent gathers sensory information which activates the simulated hippocampal place cells (CPC layer). Based on CPC activity, the AC module selects the next action.

The AC population vector $\Phi_{\mathrm{ac}}$ is calculated according to Eq. (8). It determines the allocentric direction of the next movement. The egocentric rotation angle $\theta=\Phi_{\mathrm{hd}}-\Phi_{\mathrm{ac}}$ is tied to the population vector of the head direction system (Section 3.2), which defines the allocentric angular frame of reference.

The activity of ACs is calculated in two stages. In each stage, ACs code for a different property:

Action-evaluation: First, each AC $i$ receives state information from all CPCs $j$ and learns to attribute a value to each action. This tells the agent which actions are good in the current state s. The input potential $h_{i}=\sum_{j} w_{i j} \bullet r_{j}$ to $\mathrm{ACi}$ represents the estimated value $\mathrm{Q}\left(\mathrm{s}, \mathrm{a}_{\mathrm{i}}\right)$ for the current state $s$ and action $a_{i}$. In traditional $Q$-learning, an optimal action is a discrete action whose $Q$-value is bigger or equal than all other action values. Here, we take a slightly different approach: In contrast to most other models, we do not use the max-operator to determine the optimal action. Instead, we use the direction of the AC population vector $\Phi_{\mathrm{ac}}$ (Eq. (8)). $\Phi_{\mathrm{ac}}$ represents the continuous action $a^{\mathrm{o}}$ which supposedly maximises the total future reward, given the current estimation of $Q$-values. This is called the 'greedy action' because it exploits the current estimation of $Q$-values instead of trying to improve the estimations by exploration. In order to learn all $Q$-values, we sometimes need to take non-greedy actions. This action selection method is briefly explained below. Here, we note that the optimal action $\mathrm{a}^{\mathrm{o}}$ is a continuous variable. The $Q$-values, however, are only estimated for the discrete set of ACs, i.e. $Q\left(s, a^{\mathrm{o}}\right)$ is not directly accessible. It is calculated by linear interpolation of the $Q$-values of the two nearest discrete actions.

Generalisation: As soon as an action is selected, a generalisation mechanism is applied: A Gaussian AC activity profile with standard deviation $\sigma=30^{\circ}$ is enforced around the selected action $\mathrm{a}^{\mathrm{x}}$ (see Eq. (5)). The firing rates $r_{i}$ of the $\mathrm{AC}$ layer then represent the action which was selected for execution. Recurrent connections within nucleus accumbens or via another population could be responsible for the formation of this 'blob' of activity. Traditional neural implementations of RL employ a winnertake-all mechanism which inhibits all nonselected actions. Only the winner neuron 


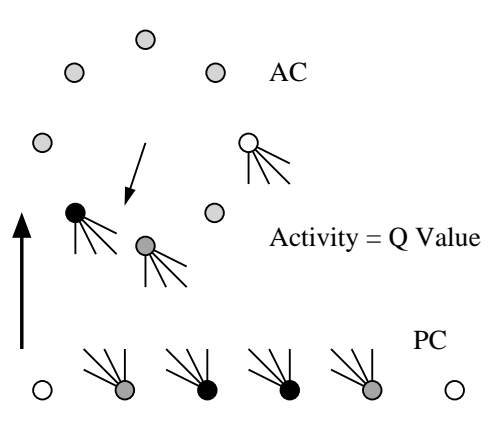

(a)

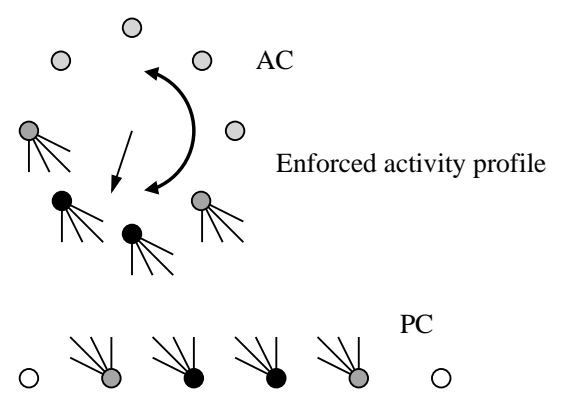

(b)

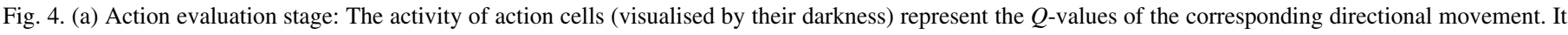

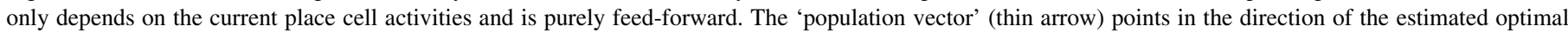

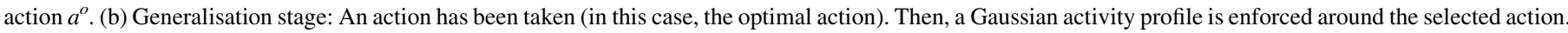
This profile allows not only the selected-but also similar actions to learn and forms the basis of the generalisation capability in action space.

may refine the estimation of its $Q$-value. In our model, however, many action cells are active and thus eligible for learning. This results in a generalisation of learning in action space.

Fig. 4 illustrates the two-stage process. In the evaluation stage, which is a purely feed-forward operation, only the current place cell activities drive ACs. The current estimation of action values, stored in the synaptic efficacies of the $\mathrm{CPC} \rightarrow \mathrm{AC}$ connections, supports the selection of an appropriate next action. Once this decision is taken, lateral interactions become effective and drive ACs above firing threshold. In the example, the 'greedy' action is taken.

The separation of the two stages of AC processing is subject to the following constraints: First, an action selection system has to be given enough time to read the optimal action $\Phi_{\mathrm{ac}}$ in the evaluation stage. Secondly, in an implementation of the learning rule with spiking neurons, the firing of ACs must closely follow action potentials emitted by CPCs such that the pre- and postsynaptic spikes fall into the timing window of spike timing dependent plasticity.

These constraints require a precise timing of the system. It has been suggested that different phases of theta activate different processing stages in hippocampus (Hasselmo, Bodelon, \& Wyble, 2002; Koene, Gorchetchnikov, Cannon, \& Hasselmo, 2003). Similarly, we propose that the theta-rhythm could provide a separation of the two stages in nucleus accumbens: First, during the late phase, low theta-activity allows hippocampal place cells to fire and thus pass spatial information to nucleus accumbens. The pathway of a recurrent loop within NA or involving another area is disabled at this time, so as not to interfere with the estimation of $Q$-values. At a later phase, when an action is selected, the recurrent loop shapes the activity profile of the generalisation stage.

\subsection{Learning algorithm}

As in all temporal-difference learning rules, the environment must be sensed before and after each action in order to update the estimation of value functions. These sensory input values stem from two adjacent time steps. Here we present the algorithm from the beginning of a learning iteration to its end, not from one time step to the next:

First, the action values $\mathrm{Q}\left(s(t-1), a_{i}\right)$, i.e. the input potentials $h_{i}$ to all action cells $i$ are calculated. Next, a continuous action $a^{\mathrm{x}}(t-1)$ is selected. Most of the time, the optimal action $a^{\mathrm{o}}(t-1)$, i.e. $\Phi_{\mathrm{ac}}$, is chosen. With a small probability $\varepsilon=0.2$, however, the new direction of movement is drawn randomly. This $\varepsilon$-greedy policy balances exploration versus exploitation (Sutton, \& Barto, 1998). The action selection process is assumed to operate on a slower time scale than the processing of sensory input. The decision to either explore or exploit is only taken every fourth time step, i.e. twice per second for a theta rhythm of $8 \mathrm{~Hz}$. Then, the AC activity profile is enforced around the selected action $a^{\mathrm{x}}(t-1)$ and the eligibility trace $e_{i j}(t-1)$ is updated. Here, the time step ends $(t-1 \rightarrow t)$. In the beginning of the new time step, the agent receives the immediate reward $\mathrm{R}(t)$. The agent processes its input, i.e. the place cell activities and $h_{i}(t)$ are updated. The standard reward prediction error $\delta(t)$ for $Q$-learning is calculated. Finally, the $\mathrm{CPC} \rightarrow \mathrm{AC}$ synaptic weights $w_{i j}$ from place cells $j$ to action cells $i$ are modified using the standard RL update rule with learning rate $\eta=$ 0.001.The following list briefly summarises these steps:

(1) Calculate action values: $\mathrm{Q}\left(s(t), \mathrm{a}_{i}\right)=h_{i}(t)$

(2) Select action: $a^{\mathrm{x}}(t)=a^{\mathrm{O}}(t)$ with probability $1-\varepsilon$ (exploitation) or randomly select action with probability $\varepsilon$ (exploration).

(3) Generalise in action space: Lateral connections impose activity profile $r_{i}(t)$ around the selected action $a^{x}(t)$.

(4) Update eligibility trace $e_{i j}(t)$.

(5) $t \rightarrow t+1$.Calculate $h_{i}(t+1)$.

(6) Calculate reward prediction error: $\delta(t+1)=R(t+1)+\gamma \cdot Q\left(s(t+1), \quad a^{o}(t+1)\right)-Q(s(t)$, $\left.a^{x}(t)\right)$.

(7) Update synaptic strengths: $\Delta w_{i j}(t+1)=\eta \cdot \delta(t+1) \cdot e_{i j}(t)$.

One problem of RL is 'the curse of dimensionality'. When the state - and action spaces are large, learning all parameters is very slow. In our case, we have $\approx 1000$ place cells and $\approx 100$ 
action cells. However, these variables are not uncorrelated. Due to the high overlap both in place and action cells, learning quickly generalises in both spaces. The size of a place cell's receptive field has a physical meaning (a portion of the environment). Similarly, the enforced AC activity profile's width $\sigma$ represents a range of headings. Both sizes are independent of the number of cells, and therefore, the learning speed is also independent of the number of cells.

\section{Experiments and results}

All experiments are conducted in the setups described in Section 2. In each setup, two stages of the experiment can be distinguished: First, the agent explores the environment and establishes the space code in the manner described in Section 3. Afterwards, the agent learns to find a hidden goal location according to Section 4.

The agent's movement consists of two primitives: (i) an inplace rotation, the angle of which depends on the current stage of the experiment and (ii) a forward movement of a fixed distance $d$ or until an obstacle is blocking the way. The step size $d$ is chosen so as to emulate a running rat: The rate at which the rat processes spatial information is assumed to be related to the theta rhythm $(\approx 8 \mathrm{~Hz})$. At each cycle, the rat 'senses' the world and reacts appropriately. We interpret one time step of the model as one theta cycle. For a constant running speed of $48 \mathrm{~cm} / \mathrm{s}$, we get $d=48 / 8=6 \mathrm{~cm}$ (Arleo \& Gerstner, 2000b; Burgess et al., 1994; Sharp, 1991; Tchernichovski, Benjamini, \& Golani, 1998).

\subsection{Exploration}

In each setup, the agent starts by exploring the environment from an arbitrary location. In each time step, the agent takes a visual snapshot and applies the processing described in Section 3. It thus recruits cells and adapts the neurons' tuning curves. The agent then turns and moves a step forward. The rotation angle is drawn from a uniform distribution between $\pm 90^{\circ}$. The agent continues exploration until the place cells densely cover the entire environment, which takes approximately 1000 time steps ( $\approx 2 \mathrm{~min}$ ). Unfortunately, completeness of exploration is difficult to define in rodents as well as in models, so we cannot compare the exploration time of our model to animal experiments. After exploration, 50 randomly chosen neurons of each the APC and the CPC population are selected and their receptive fields are measured as follows: The environment is covered by grid of $10 \times 10$ points such that the sampling points are evenly spaced. At each sampling point, the agent takes eight visual snap-shots with evenly distributed headings.

All receptive fields (RFs) have been visually inspected. In Fig. 5, the RFs of a typical APC and CPC are visualised. They are taken in the 'Buildings' environment (cf. Section 2). A cell's firing rate $r$ in the contour-plots is coded by darkness. For instance, black corresponds to $r=1.0$ and white means $r=0.0$. For each cell, a block of nine contour plots is shown. Each of the small squares represents the environment. The eight peripheral images illustrate the receptive field when the agent is oriented towards the corresponding direction. For instance, the top-right image shows the receptive field when the agent is facing northeast. The central image is the average of all directional plots. RFs of APC neurons are rather stereotype. The cells are broadly tuned around their preferred position. All APC cells observed are directional, and they are all activated in a range of about $180^{\circ}$ around the preferred agent heading. Cells that code for positions near the corners tend to have smaller RFs. In contrast, the majority of combined place cells are heading independent. Place fields are more compact than in the APC layer. Around 20\% of the observed cells are slightly directional. We observed only one cell which does not respond to all headings.

\subsection{Calibration}

In this experiment, we check whether or not the model can compensate for errors in the odometric system. Noise is

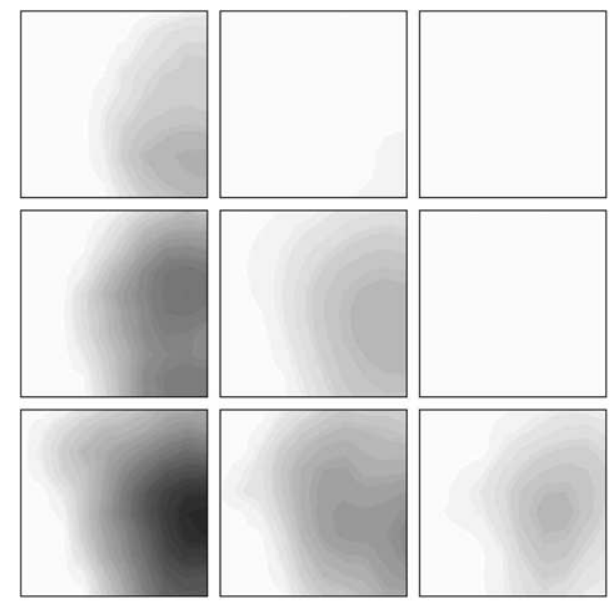

(a)
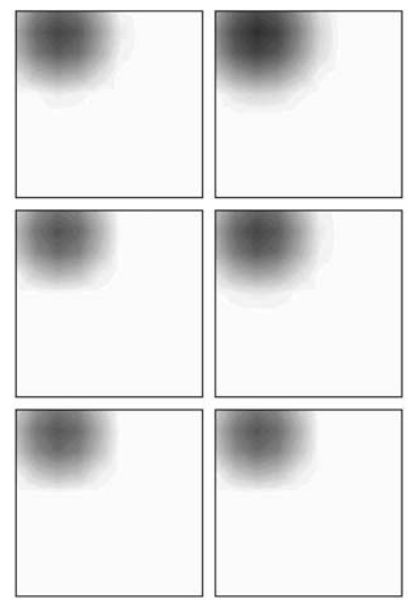

(b)

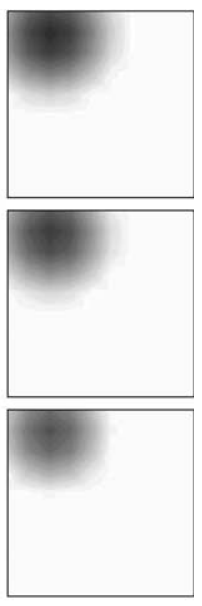

Fig. 5. Directional receptive field (RF) contour plots of (a) a typical allothetic place cell (APC) and (b) a typical combined place cell (CPC). All APCs show place and direction sensitivity and have large RFs. CPCs, in contrast are more narrowly tuned around their preferred location and are omnidirectional. 

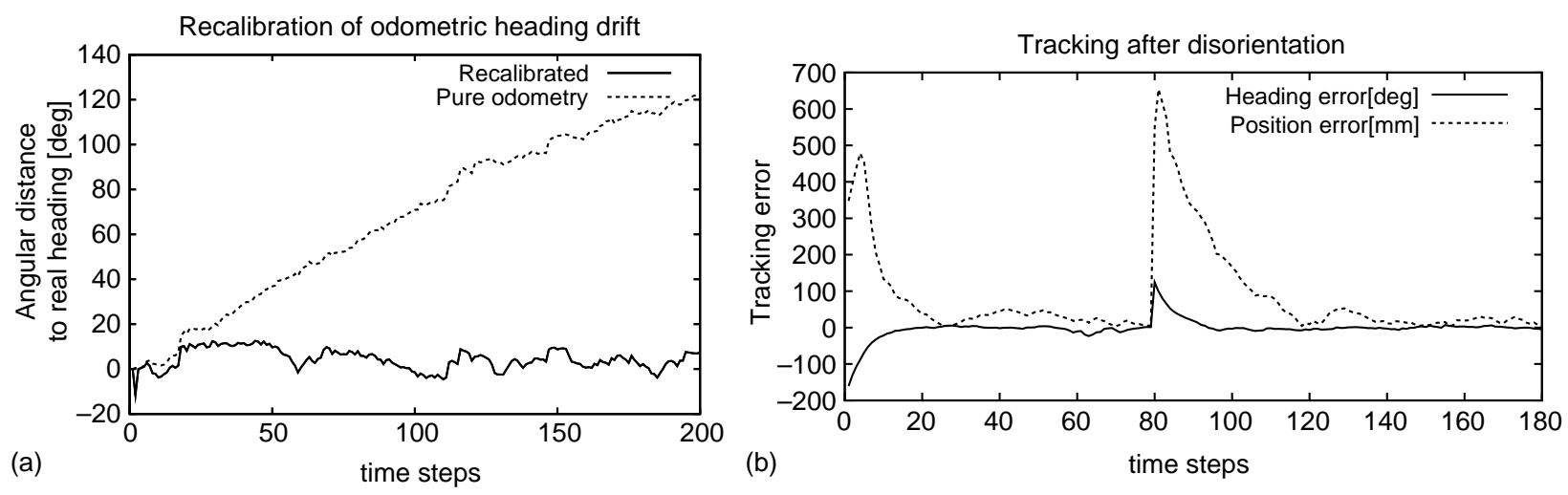

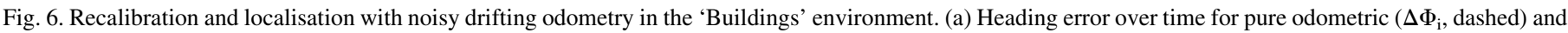

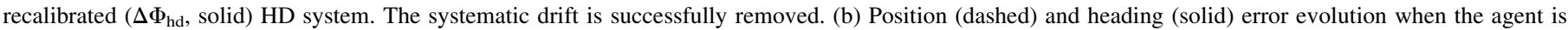
disoriented and inserted into the explored environment. At time step 80, the agent is again disoriented. The agent quickly localises itself.

artificially introduced in the simulated environments or the tracker turned-off for the real robotic setup. In simulation, we add Gaussian noise and a constant drift whenever the robot turns or moves forward.

First, we put the agent in the explored environment and initialise the path integrator (HD and PI cell populations) to the real position and heading. The agent then moves randomly for 200 time steps, applying the calibration mechanism described above (Eqs. (9) and (14)). An error histogram is computed both for the allothetic heading and position estimates $\Phi_{\mathrm{a}}$ and $\mathbf{P}_{\mathrm{a}}$. In all setups, the mean heading errors $\left\langle\Phi_{\mathrm{a}}-\Phi\right\rangle$ are below $1^{\circ}$ and the mean position errors $\left\|\left\langle\mathbf{P}_{\mathrm{a}}-\mathbf{P}\right\rangle\right\|_{2}$ are below $1 \mathrm{~cm}$.

Fig. 6(a) illustrates how the errors of the heading estimations evolve in the 'Buildings' environment. The raw odometric error (dashed) grows, as expected, linearly with time whereas the error in the HD system (solid) remains constant and thus effectively removes the drift. The position error behaves similarly. The same effects are observed in all setups.

Fig. 6(b) illustrates that the agent can not only keep its error estimation low, but it is also capable of localising itself if it is completely disoriented. The same procedure than above is applied, but this time, the agent is 'disoriented' by initialising the path integrator to a random position and heading. At time step 80, the agent is again disoriented. Both position (dashed) and heading errors (solid) are quickly reduced as the agent moves.

\subsection{Locale navigation}

Here we show that our reinforcement-based learning mechanism between place and action cells can learn to directly navigate to an invisible goal location. This is similar to the Morris water maze task with a hidden platform (Morris, 1981). As in animal experiments, the agent first explores the environment in the manner described above. During this period, no reward is given. Once the environment is fully explored, i.e. place cells densely cover the whole surface, a set of reward training trials follows.

An invisible reward is placed at a fixed location. At the beginning of each learning trial, the agent is inserted into the environment at a random location and with random heading. At each time step, the agent then executes a movement action. We use an $\varepsilon$-greedy policy: Every fourth time step, either exploitation (probability $1-\varepsilon$ ) or exploration (probability $\varepsilon$ ) is chosen. This decision is adhered to for the following three time steps. When exploitation is chosen, the agent follows the optional action $a^{\mathrm{o}}$. When exploration is chosen, however, the new direction is drawn from a Gaussian
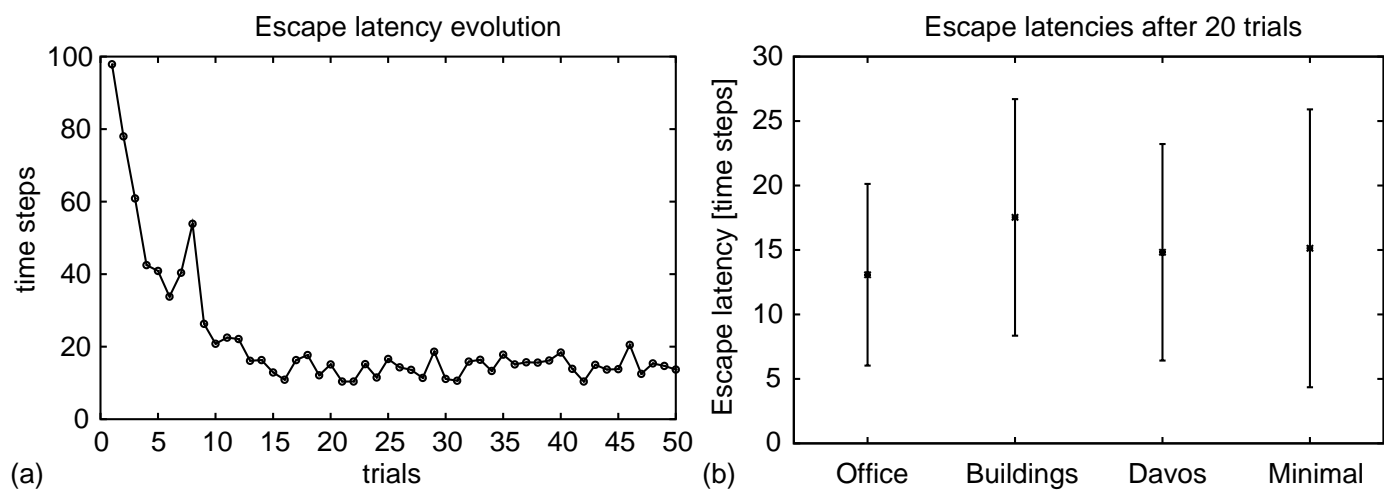

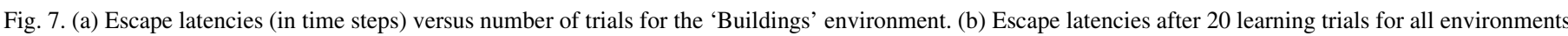

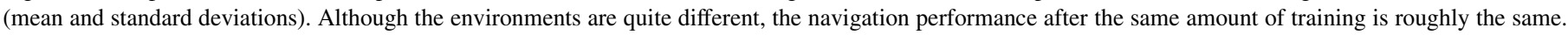




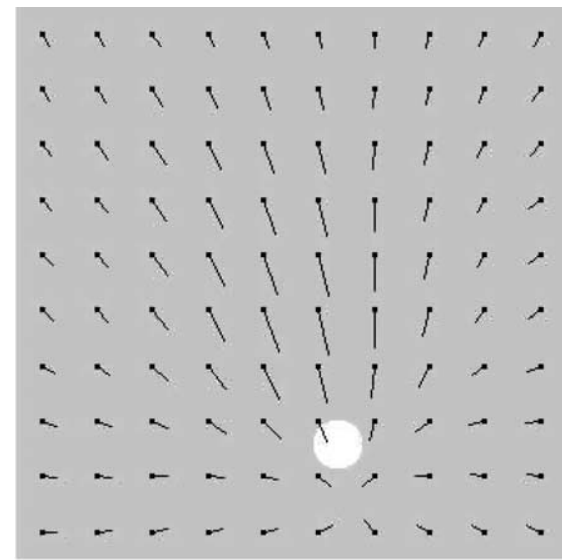

(a)

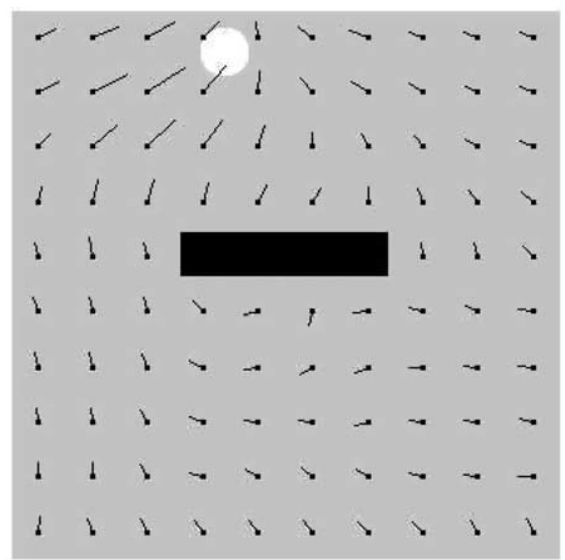

(b)

Fig. 8. Navigation maps. At each sample location marked with a black dot, a line points in the direction of the optimal action. The white area is the goal-location. (a) Navigation map acquired after 20 trials for the 'Buildings' environment. (b) An obstacle (black square) is put into the 'Buildings' environment. After 20 trials, the agent has learnt to avoid the object.

distribution with standard deviation $\sigma=30^{\circ}$ around the current heading. Whenever the agent encounters an obstacle, an immediate negative reward is administered. A trial ends when the agent finds the goal location, where a positive reward is given. During these trials, the agent adapts its $\mathrm{CPC} \rightarrow \mathrm{AC}$ synaptic strengths and thus learns a navigation map. After each learning trial, a test trial follows. The only difference between training and test trials is that learning is turned-off during test trials and the number of time steps is measured until the agent reaches the goal location. This 'escape latency' is an indication of how well the task has been acquired. In Fig. 7(a), the escape latencies versus the number of learning trials in the 'Buildings' environment are shown. After about 20 trials, the task is learnt. Similar results are obtained in the other setups. This performance is comparable to rats in the reference memory water maze task (Foster et al., 2000).

After 20 learning trials, the escape latencies are compared for all setups. Learning is disabled and 50 test trials with different starting points are performed in each environment. In Fig. 7(b), we see that the mean escape latencies are around 15 time steps for all environments. This corresponds to more or less direct paths from the starting locations. The high fluctuations reflect the variability in the start-goal distances. Furthermore, the $\varepsilon$-greedy policy adds additional noise to the escape latency.

Once the task is acquired, the agent is capable of directly navigating to the goal location from any point in the environment. This capability can be shown in a 'navigation map'. This map visualises for all locations in which direction the agent would move in order to reach the goal. Navigation maps are measured as follows: The agent is placed at a set of sampling positions which are arranged in a grid. At each such location, the agent localises itself (recalibrates the path integrator) and estimates the optimal heading $\Phi_{\mathrm{ac}}$.

Fig. 8(a) is the navigation map measured after 20 learning trials in the 'Buildings' environment. The sampling points are indicated as black dots A line is drawn from each sampling position in the direction of $\Phi_{\mathrm{ac}}$. The white area represents the goal location. The agent has indeed learnt to directly navigate to the target location. However, the navigation map contains some errors in the region below the goal. One reason is that the starting points of all trials have been set at a minimum distance of $20 \mathrm{~cm}$. This means that the agent never starts beneath the goal. In general, the probability that the agent is located in a central position is higher than for locations near the walls. Therefore, the agent approaches the target more often from the north than from the south. As a consequence, the estimates of Q-values are better for central locations because they have been adapted more often. Secondly, the generalisation in state space, i.e. the large tuning curves of place cells, falsely reinforces the 'go-south' actions in places to the south of the goal even when reaching the goal from the north. A possible solution would be to give stronger negative reward when a wall is hit. However, this would make learning goal locations near obstacles difficult.

Finally, we also test if a usable navigation map can be learnt in the presence of local obstacles. We therefore run a simulation in the 'Buildings' environment with a rectangular obstacle blocking the centre of the arena. After full exploration, the agent receives 20 training trials. The obtained navigation map is shown in Fig. 8(b). Indeed, the agent learns to avoid the obstacle and find a path leading to the goal location from any location. However, the path is not always the shortest. In this example, the agent prefers avoiding the rectangle to its left. The reason for this asymmetry lies in the coupling of the action value estimation and the policy. As soon as one path is successful, the sampling of the environment becomes biased. This problem is related to the exploration-exploitation tradeoff. A possible solution could be to use an $\varepsilon$-greedy policy with variable $\varepsilon$. However, finding a rule which chooses the right policy for all tasks is still an open problem.

\section{Discussion}

The model presented here is capable of constructing and maintaining a consistent place code. It is shown how such a 
spatial representation can be used to solve the hidden platform Morris water-maze task. Although comparable to previous work, this proposal mainly adds the following contributions: (i) real-istic input. This model relies on real visual input, just like (Arleo, Smeraldi, \& Gerstner, 2004; Gaussier et al., 2000). However, our system does not need compass nor polarising cue in order to maintain a stable representation. (ii) recalibration. We show how a drifting path integrator can be recalibrated using allothetic information. Although there are other models which perform recalibration, our proposal has several advantages: Firstly, this simple mechanism, unlike Bayesian methods (Herrmann et al., 1999), can easily be implemented by neural systems. Unlike (Arleo, \& Gerstner, 2000b), neither receptive field nor place code quality information is needed for recalibration. Secondly, the computational cost and convergence speed of our mechanism is much lower than Bayesian methods (Herrmann et al., 1999). Lastly, our method is not restricted to particular place field shapes (Deneve et al., 2001). (iii)Navigation in continuous space. Reward-based learning, as used in (Arleo, \& Gerstner, 2000b; Brown, \& Sharp, 1995; Burgess et al., 1994; Foster et al., 2000; Gaussier et al., 2002), does not scale well to bigger state and action spaces. Additionally, actions are represented by a small discrete set of neurons. Our model is continuous in both spaces and features a generalisation mechanism. Therefore, the learning speed is independent of the number of used neurons. Unlike (Brown, \& Sharp, 1995; Burgess et al., 1994), a distal reward problem is avoided by using a temporal-difference reinforcement learning algorithm. Other models (Blum, \& Abbott, 1996; Redish, \& Touretzky, 1998) also operate in continuous space. However, they model locale navigation inside the hippocampus, which contradicts experimental data (Eichenbaum et al., 1990; Packard, \& McGaugh, 1992; Sutherland, \& Rodriguez, 1990).

The results of the present study yields a number of predictions. These could help designing new experiments which in turn could confirm or disprove the proposed model. Previous results show that postsubiculum (poSb) contains head direction cells (HD) (Ranck, 1984; Taube et al., 1990). Similar to others (Arleo, \& Gerstner, 2001; Redish, \& Touretzky, 1997a), we suggest that poSb forms the output stage of a head direction system which combines an internal heading integrator with visual input in order to remove a heading drift. Our work, similar to (Arleo, \& Gerstner, 2000b) proposes the lateral entorhinal cortex (IEC) as neural substrate for a purely allothetic place code. Consequently, poSb lesions should not affect IEC place fields. In case of conflicting idiothetic and allothetic cues, IEC cells should follow the allothetic cue. Furthermore, we predict that place cells in IEC are directional. Similar to (Arleo, \& Gerstner, 2000b; Redish, \& Touretzky, 1997a) and contrary to (McNaughton et al., 1996; Samsonovich, \& McNaughton, 1997), we suggest that the medial entorhinal cortex (mEC) and possibly the subiculum ( $\mathrm{Sb}$ ) may form the neural substrate for a position integrator. Lesions in poSb or its afferent structures, which may provide mEC with the animal's heading, should produce severe inconsistencies in $\mathrm{mEC}$. Indeed, it has been shown that such lesions produce severe behavioural deficits
(Taube, Klessak, \& Cotman, 1992). Furthermore, mEC place fields are predicted to be non-directional even when the animal's movement is restricted. Disrupting the allothetic pathway and in particular lesioning $\mathrm{lEC} \rightarrow \mathrm{mEC}$ connections should produce a drifting position integrator. In our model, position integration in $\mathrm{mEC}$ and a visual place code in IEC combine into a combined spatial map in the hippocampal region $\mathrm{CA} 1$ and possibly $\mathrm{Sb}$. Lesions in mEC should leave CA1 with more broadly tuned, purely allothetic, directional place cells. In contrast, lesions in IEC should produce omnidirectional firing in CA1 even in cases where these cells are normally directional. Lesions to the HD system should disrupt place cell firing in HPC. However, joint lesions in mEC should improve performance again (if $1 \mathrm{EC}$ is still intact) and leave the hippocampus with broadly tuned, directional, allothetic place cells.

Many important issues in hippocampal function in general and it's significance to spatial learning in particular are yet unanswered. Several experiments indicate that a wide range of species use some sort of path integration (Etienne, Boulens, Maurer, Rowe, \& Siegrist, 2000; Wehner, 2003). Despite considerable effort, however, little is known about the underlying mechanisms. Experimental data show that hippocampus and fornix lesions disrupt path integration (Maaswinkel, Jarrard, \& Whishaw, 1999; Whishaw, \& Maaswinkel, 1998). However, other data also suggests that path integration is possible without the hippocampus proper (Alyan, \& McNaughton, 1999; Alyan, Paul, Ellesworth, White, \& McNaughton, 1997). It is still unclear how these experimental results can be reconciled. Place cells are strongly influenced by vision. However, somatosensory, olfactive and internal (self-motion) cues seem to contribute to the formation and maintenance of place fields (Etienne et al., 2000; Lavenex, \& Schenk, 1998; Markus et al., 1994; Quirk et al., 1990; Save et al., 2000). It is not yet clear how these different modalities are integrated into one stable representation. This model could learn to represent several environments. New place cells would have to be recruited. Rats can, however, reuse place cells in several environments. Their place fields in those environments are totally unrelated (Kubie, \& Ranck, 1983; Thompson, \& Best, 1989). We are not aware of any biologically plausible place cell model which (i) depends on path integration, (ii) can code for several environments and (iii) features totally unrelated place fields in those environments. Indeed, the underlying principle of these effects are still open questions.

\section{References}

Alyan, S., \& McNaughton, B. L. (1999). Hippocampectomized rats are capable of homing by path integration. Behavioral Neuroscience, 113(1), 19-31.

Alyan, S. H., Paul, B. M., Ellesworth, E., White, R. D., \& McNaughton, B. L. (1997). Is the hippocampus required for path integration? Society for Neuroscience Abstracts, 23, 504.

Amaral, D. G., \& Witter, M. P. (1989). The three-dimensional organization of the hippocampal formation: A review of anatomical data. Neuroscience, 31(3), 571-591.

Amaral, D. G., \& Witter, M. P. (1995). Hippocampal formation. In G. Paxinos (Ed.), The Rat Nervous System (2nd ed.) (pp. 443-493). Academic Press (Chapter 21). 
Amari, S.-I. (1977). Dynamics of pattern formation in lateral-inhibition type neural fields. Biological Cybernetics, 27, 77-87.

Arleo, A., \& Gerstner, W. (2000a). Modeling rodent head-direction cells and place cells for spatial learning in bio-mimetic robotics. In J.-A. Meyer, A. Berthoz, D. Floreano, H. L. Roitblat, \& S. W. Wilson (Eds.), From Animals to Animats VI (pp. 236-245). Cambridge MA: MIT Press.

Arleo, A., \& Gerstner, W. (2000b). Spatial cognition and neuro-mimetic navigation: A model of hippocampal place cell activity. Biological Cybernetics, Special Issue on Navigation in Biological and Artificial Systems, 83, 287-299.

Arleo, A., \& Gerstner, W. (2001). Spatial orientation in navigating agents: Modeling head-direction cells. Neurocomputing, 38(40), 1059-1065.

Arleo, A., Smeraldi, F., \& Gerstner, W. (2004). Cognitive navigation based on nonuniform gabor space sampling, unsupervised growing networks, and reinforcement learning. IEEE Transactions on Neural Networks, 15(3), 639-652.

Arleo, A., Smeraldi, F., Hug, S., \& Gerstner, W. (2001). Place cells and spatial navigation based on $2 \mathrm{~d}$ visual feature extraction, path integration, and reinforcement learning. In T. K. Leen, T. G. Dietterich, \& V. Tresp (Eds.), Advances in neural information processing systems 13 (pp. 89-95). Cambridge MA: MIT Press.

Blum, K. I., \& Abbott, L. F. (1996). A model of spatial map formation in the hippocampus of the rat. Neural Computation, 8, 85-93.

Boucheny, C., Brunel, N., \& Arleo, A. (2005). A continuous attractor network model without recurrent excitation: Maintenance and integration in the head direction cell system. Journal of Computational Neuroscience, 18(2), 205-227.

Brown, M. A., \& Sharp, P. E. (1995). Simulation of spatial-learning in the Morris water maze by a neural network model of the hippocampalformation and nucleus accumbens. Hippocampus, 5, 171-188.

Burgess, N., Donnett, J. G., Jeffery, K. J., \& O'Keefe, J. (1997). Robotic and neuronal simulation of the hippocampus and rat navigation. Philosophical Transactions of the Royal Society London B, 352, 1535-1543.

Burgess, N., Jackson, A., Hartley, T., \& O'Keefe, J. (2000). Predictions derived from modelling the hippocampal role in navigation. Biological Cybernetics, 83, 301-312.

Burgess, N., Recce, M., \& O'Keefe, J. (1994). A model of hippocampal function. Neure Networks, 7, 1065-1081.

Buzsáki, G. (1984). Feed-forward inhibition in the hippocampal formation. Progress in Neurobiology, 22, 131-153.

Deneve, S., Latham, P. E., \& Pouget, A. (2001). Efficient computation and cue integration with noisy population codes. Nature Neuroscience, 4(8), 826831.

Eichenbaum, H., Stewart, C., \& Morris, R. G. M. (1990). Hippocampal representation in place learning. Journal of Neuroscience, 10(11), 35313542.

Etienne, A. S., Boulens, V., Maurer, R., Rowe, T., \& Siegrist, C. (2000). A brief view of known landmarks reorientates path integration in hamsters. Naturwissenschaften, 87(11), 494-498.

Foster, D. J., Morris, R. G. M., \& Dayan, P. (2000). A model of hippocampally dependent navigation, using the temporal difference learning rule. Hippocampus, 10(1), 1-16.

Freund, T. F., Powell, J. F., \& Smith, A. D. (1984). Tyrosine hydroxylaseimmunoreactive boutons in synaptic contact with identified striatonigral neurons, with particular reference to dendritic spines. Neuroscience, 13(4), $1189-1215$.

Gabor, D. (1946). Theory of communication. Journal of the IEE, 93, 429-457.

Gaussier, P., Joulain, C., Banquet, J. P., Leprêre, S., \& Revel, A. (2000). The visual homing problem: An example of robotics/biology cross fertilization. Robotics and Autonomous Systems, 30(1-2), 155-180.

Gaussier, P., Leprêtre, S., Joulain, C., Revel, A. Quoy, M., \& Banquet J. P. (1998). Animal and robot learning: Experiments and models about visual navigation. In 7th European Workshop on Learning Robots, Edinburgh, $U K$.

Gaussier, P., Revel, A., Banquet, J. P., \& Babeau, V. (2002). From view cells and place cells to cognitive map learning: Processing stages of the hippocampal system. Biological Cybernetics, 86(1), 15-28.
Georgopoulos, A. P., Schwartz, A., \& Kettner, L. E. (1986). Neuronal population coding of movement direction. Science, 233, 1416-1419.

Gerstner, W., \& Abbott, L. F. (1997). Learning navigational maps through potentiation and modulation of hippocampal place cells. Journal of Computational Neuroscience, 4(1), 79-94.

Hartley, T., Burgess, N., Lever, C., Cacucci, F, \& O'Keefe, J. (2000). Modeling place fields in terms of the cortical inputs to the hippocampus. Hippocampus, 10, 369-379.

Hasselmo, M. E., Bodelon, C., \& Wyble, B. P. (2002). A proposed function for hippocampal theta rhythm: Separate phases of encoding and retrieval enhance reversal of prior learning. Neural Computer, 14(4), 793-817.

Hasselmo, M. E., \& Bower, J. M. (1993). Acetylcholine and memory. Trends in Neurosciences, 16(6), 218-222.

Herrmann, J. M., Pawelzik, K., \& Geisel, T. (1999). Self-localization of autonomous robots by hidden representations. Autonomous Robots, 7(1), $31-40$.

Hubel, D. H., \& Wiesel, T. N. (1962). Receptive fields, binocular interaction and functional architecture in the cat's visual cortex. Journal of Physiology, 160, 106-154.

Hughes, A. (1977). The topography of visuon in mammals of contrasting life style: Comparative optics and retinal organisation. In F. Crescitelli, The visual system in vertebrates, Handbook of sensory physiology (Vol. 7/5) (pp. 613-756). Berlin: Springer-Verlag (Chapter 11).

Jeffery, K. J. (Ed.). (2003). The neurobiology of spatial behavior. Oxford University Press.

Knierim, J. J., Kudrimoti, H. S., \& McNaughton, B. L. (1995). Place cells, head direction cells, and the learning of landmark stability. Journal of Neuroscience, 15, 1648-1659.

Koene, R. A., Gorchetchnikov, A., Cannon, R. C., \& Hasselmo, M. E. (2003). Modeling goal-directed spatial navigation in the rat based on physiological data from the hippocampal formation. Neural Networks, 16(5-6), 577-584.

Kubie, J. L., \& Ranck, J. B. (1983). Sensory-behavioral correlates in individual hippocampus neurons in three situations: Space and context. In W. Seifert (Ed.), Neurobiology of the Hippocampus (pp. 433-447). New York: Academic Press.

Lavenex, P., \& Schenk, F. (1998). Olfactory traces and spatial learning in rats. Animal Behaviour, 56, 1129-1136.

Maaswinkel, H., Jarrard, L. E., \& Whishaw, I. Q. (1999). Hippocampectomized rats are impaired in homing by path integration. Hippocampus, 9(5), 553561.

Markus, E. J., Barnes, C. A., McNaughto, B. L., Gladden, V. L., \& Skaggs, W. E. (1994). Spatial information content and reliability of hippocampal cal neurons: Effects of visual input. Hippocampus, 4, 410-421.

McNaughton, B. L., Barnes, C. A., Gerrard, J. L., Gothard, K., Jung, M. W., Knierim, J. J., et al. (1996). Deciphering the hippocampal polyglot: the hippocampus as a path integration system. Journal of Experimental Biology, 199(1), 173-185.

McNaughton, B. L., Barnes, C. A., \& O'Keefe, J. (1983). The contributions of position, direction, and velocity to single unit activity in the hippocampus of freely-moving rats. Experimental Brain Research, 52, 41-49.

Miller, R. (1991). Cortico-hippocampal interplay and the representation of contexts in the brain. Berlin: Springer.

Mondada, F., Franzi, E., \& Ienne, P. (1994). Mobile robot miniaturization: A tool for investigation in control algorithms. In T. Yoshikawa, \& F. Miyazaki (Eds.), Proceedings of the third international symposium on experimental robotics (pp. 501-513). Tokyo: Springer.

Morris, R. G. M. (1981). Spatial localization does not require the presence of local cues. Learning and Motivation, 12, 239-260.

Muller, R. U., Bostock, E., Taube, J. S., \& Kubie, J. L. (1994). On the directional firing properties of hippocampal place cells. Journal of Neuroscience, 14(12), 7235-7251.

Muller, R. U., \& Kubie, J. L. (1987). The effects of changes in the environment on the spatial firing of hippocampal complex-spike cells. Journal of Neuroscience, 7, 1951-1968.

O'Keefe, J., \& Burgess, N. (1996). Geometric determinants of the place fields of hippocampal neurons. Nature, 381, 425-428. 
O'Keefe, J., \& Dostrovsky, J. (1971). The hippocampus as a spatial map. Preliminary evidence from unit activity in the freely-moving rat. Brain Research, 34, 171-175.

O'Keefe, J., \& Nadel, L. (1978). The Hippocampus as a cognitive map. Oxford: Clarendon Press.

O'Keefe, J., \& Recce, M. (1993). Phase relationship between hippocampal place units and the EEG theta rhythm. Hippocampus, 3, 317-330.

Otto, T., Ding, C., Cousens, G., \& Schiller, K. (1996). Effects of lateral vs. medial entorhinal cortex aspiration on the acquisition of odor-place associations. Society for Neuroscience Abstracts, 22, 1120.

Packard, M. G., \& McGaugh, L. (1992). Double dissociation of fornix and caudate nucleus lesions on acquisition of two water maze tasks: Further evidence for multiple memory systems. Behavioral Neuroscience, 106(3), 439-446.

Pouget, A., Deneve, S., \& Duhamel, J. R. (2002). A computational perspective on the neural basis of multisensory spatial representations. Nature Review Neuroscience, 3(9), 741-747.

Quirk, G. J., Muller, R. U., \& Kubie, J. L. (1990). The firing of hippocampal place cells in the dark depends on the rat's recent experience. Journal of Neuroscience, 10(6), 2008-2017.

Quirk, G. J., Muller, R. U., Kubie, J. L., \& Ranck, J. B. Jr. (1992). The positional firing properties of medial entorhinal neurons: Description and comparison with hippocampal place cells. Journal of Neuroscience, 12(5), 1945-1963.

Ranck, J. B. Jr. (1984). Head-direction cells in the deep cell layers of dorsal presubiculum in freely moving rats. Society for Neuroscience Abstracts, 10 , 599.

Redish, A. D. (1999). Beyond the cognitive map, from place cells to episodic memory. London: MIT Press-Bradford Books.

Redish, A. D., \& Touretzky, D. S. (1997a). Cognitive maps beyond the hippocampus. Hippocampus, 7(1), 15-35.

Redish, A. D., \& Touretzky, D. S. (1997b). Navigating with landmarks: Computing goal locations from place codes. In K. Ikeuchi, \& M. Veloso (Eds.), Symbolic visual learning (pp. 325-351). Oxford: Oxford University Press (Chapter 12).

Redish, A. D., \& Touretzky, D. S. (1998). The role of the hippocampus in solving the morris water maze. Neural Computation, 10(1), 73-111.

Salinas, E., \& Abbott, L. F. (1994). Vector reconstruction from firing rates. Journal of Computational Neuroscience, 1, 89-107.

Samsonovich, A., \& McNaughton, B. L. (1997). Path integration and cognitive mapping in a continuous attractor neural network model. Journal of Neuroscience, 17(15), 5900-5920.

Sanger, T. D. (2003). Neural population codes. Current Opinion Neurobiology, 13(2), 238-249.

Save, E., Nerad, L., \& Poucet, B. (2000). Contribution of multiple sensory information to place field stability in hippocampal place cells. Hippocampus, 10, 64-76.

Schultz, W. (1998). Predictive reward signal of dopamine neurons. Journal of Neurophysiology, 80, 1-27.
Schultz, W., Dayan, P., \& Montague, R. P. (1997). A neural substrate of prediction and reward. Science, 275, 1593-1599.

Sesack, S. R., \& Pickel, V. M. (1990). In the rat medial nucleus accumbens, hippocampal and catecholaminergic terminals converge on spiny neurons and are in apposition to each other. Brain Research, 527(2), 266-279.

Sharp, P. E. (1991). Computer simulation of hippocampal place cells. Psychobiology, 19(2), 103-115.

Skaggs, W. E., McNaughton, B. L., Wilson, M. A., \& Barnes, C. A. (1996) Theta phase precession in hippocampal neuronal populations and the compression of temporal sequences. Hippocampus, 6(2), 149-173.

Sutherland, R. J., \& Rodriguez, A. J. (1990). The role of the fornix/fimbria and some related subcortical structures in place learning and memory. Behavioral and Brain Research, 32, 265-277.

Sutton, R., \& Barto, A. G. (1998). Reinforcement learning-An introduction. MIT Press.

Taube, J. S., Klessak, J. P., \& Cotman, C. W. (1992). Lesions of the rat postsubiculum impair performance on spatial tasks. Behavioral and Neural Biology, 5, 131-143.

Taube, J. S., Muller, R. I., \& Ranck, J. B. Jr. (1990). Head direction cells recorded from the postsubiculum in freely moving rats. I. Description and quantitative analysis. Journal of Neuroscience, 10, 420-435.

Tchernichovski, O., Benjamini, Y., \& Golani, I. (1998). The dynamics of long-term exploration in the rat. Part i. A phase-plane analysis of the relationship between location and velocity. Biological Cybernetics, 78(6), 423-432.

Thompson, L. T., \& Best, P. J. (1989). Place cells and silent cells in the hippocampus of freely-behaving rats. Journal of Neuroscience, 9(7), 2382 2390.

Touretzky, D. S., \& Redish, A. D. (1996). A theory of rodent navigation based on interacting representations of space. Hippocampus, 6(3), 247-270.

Wan, H. S., Touretzky, D. S., \& Redish, A. D. (1994). Towards a computational theory of rat navigation. In M. Mozer, P. Smolensky, D. S. Touretzky, J. Elman, \& A. Weigend (Eds.), Proceedings of the 1993 connectionist models summer school (pp. 11-19). Hillsdale, NJ: Erlbaum.

Wehner, R. (2003). Desert ant navigation: how miniature brains solve complex tasks. Journal of Comparative Physiology A-Neuroethology Sensory Neural and Behavioral Physiology, 189(8), 579-588.

Whishaw, I. Q., \& Maaswinkel, H. (1998). Rats with fimbria-fornix lesions are impaired in path integration: a role for the hippocampus in sense of direction. Journal of Neuroscience, 18(8), 3050-3058.

Whishaw, I. Q., \& Mittleman, G. (1991). Hippocampal modulation of nucleus accumbens: Behavioral evidence from amphetamine-induced activity profiles. Behavioral and Neural Biology, 55, 289-306.

Winson, J. (1978). Loss of hippocampal theta rhythm results in spatial memory deficits in the rat. Science, 201(160), 163.

Zhang, K. (1996). Representation of spatial orientation by the intrinsic dynamics of the head-direction cell ensemble: A theory. Journal of Neuroscience, 16(6), 2112-2126. 